\title{
Stimulated emission of dark matter axion from condensed matter excitations
}

\author{
Naoto Yokoi $^{a}$ and Eiji Saitoh ${ }^{a, b, c}$ \\ ${ }^{a}$ Institute for Materials Research, Tohoku University, \\ Sendai 980-8577, Japan \\ ${ }^{b}$ Advanced Institute for Materials Research, Tohoku University, \\ Sendai 980-8577, Japan \\ ${ }^{c}$ Advanced Science Research Center, Japan Atomic Energy Agency, \\ Tokai 319-1195, Japan \\ E-mail: yokoi@imr.tohoku.ac.jp, eizi@imr.tohoku.ac.jp
}

ABSTRACT: We discuss a possible principle for detecting dark matter axions in galactic halos. If axions constitute a condensate in the Milky Way, stimulated emissions of the axions from a type of excitation in condensed matter can be detectable. We provide general mechanism for the dark matter emission, and, as a concrete example, an emission of dark matter axions from magnetic vortex strings in a type II superconductor is investigated along with possible experimental signatures.

Keywords: Effective Field Theories, Beyond Standard Model, Cosmology of Theories beyond the SM, Discrete Symmetries

ARXIV EPRINT: 1612.05406 


\section{Contents}

1 Introduction 1

2 Properties of dark matter axions $\quad 2$

2.1 Coupling of axion with electromagnetic field 2

2.2 Non-relativistic limit of axion field 4

2.3 Dark matter axion as condensate 5

$\begin{array}{lll}3 & \text { Excitations coupled with axions in condensed matter } & 6\end{array}$

4 Axion-vortex coupling in type II superconductors $\quad 7$

4.1 Ginzburg-Landau description of superconductor and magnetic vortex $\quad 7$

4.2 Vortex dynamics with axion coupling 8

$\begin{array}{lll}4.3 & \text { Stimulated emission of axions from vortices } & 10\end{array}$

5 Possible signature of dark matter axions $\quad 14$

5.1 Stimulated emission from other excitations in condensed matter $\quad 14$

$\begin{array}{lll}6 & \text { Summary and discussion } & 15\end{array}$

$\begin{array}{ll}\text { A Dimensional analysis on axion coupling } & 16\end{array}$

\section{Introduction}

Recent discovery of the Higgs particle revealed that the standard model of particle physics correctly describes the fundamental constituents of our universe, up to the energy scale $\sim 1 \mathrm{TeV}[1]$. However, the current standard cosmology strongly indicates the existence of additional constituents, which are known as the dark matter $[2,3]$. In particular, observations of the rotation velocities of galaxies imply that there exists the dark matter halo around the Milky Way, whose mass density is given by

$$
\rho_{\mathrm{DM}} \simeq 3 \times 10^{14}\left[\mathrm{eV} / \mathrm{c}^{2} \mathrm{~m}^{3}\right] \simeq 5 \times 10^{-22}\left[\mathrm{~kg} / \mathrm{m}^{3}\right] .
$$

Although there are various candidates of the dark matter, including the weakly interacting massive particles (WIMPs), the origin and properties of the dark matter remain a mystery, so far. (See $[4,5]$ for the current status of the dark matter research.)

Among them, the axion is one of the promising candidates for the dark matter in our universe. The axion is a hypothetical elementary particle, which gives the most plausible solution to the strong CP puzzle in quantum chromodynamics (QCD) [6, 7]. Through various experiments and astrophysical observations for axion search, the properties of the 
axion, such as the mass and coupling strength, are severely constrained $[1,8,9]$. For example, the typical mass consistent with the experimental constraints can be summarized as

$$
10^{-6}\left[\mathrm{eV} / c^{2}\right] \lesssim m_{a} \lesssim 10^{-3}\left[\mathrm{eV} / c^{2}\right]
$$

for the invisible QCD axion models [11-14]. This small mass distinguishes the axions from other WIMPs, whose masses are assumed to be $m_{\text {wimp }} \gtrsim 10^{9}\left[\mathrm{eV} / c^{2}\right]$. In contrast to the standard WIMPs which are usually fermions, axions obey the Bose-Einstein statistics. In order to consistently explain the dark matter density in terms of the axion, a large number density of the bosonic axions is required in our galaxy, and the possibility of the Bose-Einstein condensation of dark matter axions due to such a large density has been discussed [15-19].

In this paper, we discuss a possible consequence of the Bose-Einstein condensation of the dark matter axions: stimulated emissions of the axions. Based on the coupling between the axion field and the electromagnetic field, we discuss the stimulated emissions of the axions from collective excitations in various condensed matter systems. In particular, as a concrete example, we investigate the stimulated emission from the magnetic vortex strings in type II superconductors, where a mobile vortex ensemble, such as vortex flow or vortex liquid, is realized near the critical temperature. The emission rate of the dark matter axions is estimated, and possible experimental signature of the emission are discussed.

\section{Properties of dark matter axions}

In this section, we briefly summarize the fundamental properties of the axion, in particular, the interaction between the axions and the electromagnetic fields. We also discuss the properties of the axion which make it to be a good candidate for the (cold) dark matter, with an emphasis on the difference from other dark matter candidates, such as WIMPs.

\subsection{Coupling of axion with electromagnetic field}

Since the axion is a (pseudo) Nambu-Goldstone boson [20,21] of the spontaneous breaking of the Peccei-Quinn (PQ) symmetry $[6,7]$, the axion field $\phi_{a}$ couples to other particles through its derivative, originated from a universal coupling,

$$
\mathcal{L}_{\mathrm{NG}} \simeq j_{\mathrm{PQ}}^{\mu}\left(\partial_{\mu} \phi_{a}\right)
$$

where $j_{\mathrm{PQ}}^{\mu}$ is the conserved current of the PQ-symmetry. Although the strength of this coupling is very small for the invisible axion models, various experiments to detect the axions using this type of coupling have been implemented so far $[8,9]$.

The axion has an additional coupling in the leading order with the electromagnetic (EM) field, which is a parity-odd coupling originated from the quantum anomaly of the PQ symmetry:

$$
\mathcal{L}_{\text {int }}=g_{0} \phi_{a} \vec{E} \cdot \vec{B}
$$


In this paper, we focus on this axion coupling (2.2) and discuss its consequence. ${ }^{1}$ With this coupling, the action for the axion field and EM-field is given by

$$
\begin{aligned}
S= & \int d^{4} x \frac{1}{2}\left[\frac{\hbar^{2}}{c^{2}}\left(\frac{\partial \phi_{a}}{\partial t}\right)^{2}-\hbar^{2}\left(\vec{\nabla} \phi_{a}\right)^{2}-m_{a}^{2} c^{2} \phi_{a}^{2}\right] \\
& +\int d^{4} x\left[\left(\frac{\varepsilon_{0}}{2} \vec{E}^{2}-\frac{1}{2 \mu_{0}} \vec{B}^{2}\right)+g_{0} \phi_{a} \vec{E} \cdot \vec{B}+\cdots\right],
\end{aligned}
$$

where $m_{a}$ is the mass of the axion and the dots represent higher order terms with respect to the coupling $g_{0}$. The coupling strength $g_{0}$ is constrained to be very small from various experiments and astronomical observations $[1,8,9]$, and the current bound, from the CAST experiment [10], is given by

$$
g_{0} \lesssim 0.66 \times 10^{-10}\left[\mathrm{GeV}^{-1}\right] \simeq 10^{-50}\left[\frac{\sec C^{2}}{\sqrt{\operatorname{kg} m}}\right],
$$

where the first value is in the natural units, and the second one is in the SI units with $C$ being Coulomb for the electric charge. (See the appendix for the conversion between these unit systems.) We optimistically take the value $g_{0} \simeq 0.5 \times 10^{-10}\left[\mathrm{GeV}^{-1}\right]$ in this paper, for concreteness. It should be noted that the invisible QCD axion models, such as the KSVZ model $[11,12]$ and DFSZ model $[13,14]$, typically lead to smaller couplings $g_{0} \simeq 10^{-14}\left[\mathrm{GeV}^{-1}\right]$ for the axion mass range (1.2). Our discussion is based solely on the low-energy effective action (2.3), and almost model-independent. ${ }^{2}$ In the following, we consider general axion models, including axion-like particles.

Although the coupling (2.2) leads to the axion-photon scattering and the axion decay to two photons, the scattering and decay rates are very small and the lifetime of the axion is much longer than the current age of the universe. This indicates that the axions produced in the early universe with some mechanism, such as the misalignment of the vacuum angle and the cosmic string decay, can remain for a long time, and these relic axions can be promising candidates for the dark matter in our universe. Particularly, the production mechanisms are mainly non-thermal, and the axions, which are produced around the time of the QCD transition, behave as non-relativistic particles from the beginning. So, the axion is a good candidate for the cold dark matter $[2,3]$.

If the axion accounts for the major part of the dark matter of our universe, the abundance of the axion in our galaxy should explain the mass density (1.1), which is determined by the observation of the rotation curve of various galaxies $[4,5]$. The velocity distribution of the dark matter in the galactic halo has also been discussed, and it is known that the violent relaxation by gravitational attraction [22] leads to the Maxwellian distribution, where the root-mean-squared velocity is given by

$$
v_{a} \simeq 2.7 \times 10^{5}[\mathrm{~m} / \mathrm{sec}] \sim 10^{-3} \mathrm{c} .
$$

\footnotetext{
${ }^{1}$ The experiments for the axion detection using this coupling have also been performed [8, 9].

${ }^{2}$ Due to the small coupling strength, the higher order terms with respect to $g_{0}$ can be completely neglected.
} 
As noted above, since $v_{a} / c \sim 10^{-3} \ll 1$, the axions in our galaxy behave as non-relativistic particles. Although the mass of the axion depends on the models beyond the standard model of the elementary particles, the plausible mass range is discussed from the various viewpoints including cosmology, astronomy and elementary particle physics [8]. In particular, in order to explain the dark matter abundance in our universe, the expected mass should be in the range (1.2). If we take $m_{a} \simeq 10^{-6}\left[\mathrm{eV} / c^{2}\right]$ as a typical value, we can calculate the number density $n_{a}$ of the dark matter axions in our galaxy,

$$
n_{a}=\frac{\rho_{\mathrm{DM}}}{m_{a}} \simeq 3 \times 10^{20}\left[m^{-3}\right] .
$$

\subsection{Non-relativistic limit of axion field}

Since the axions behave as non-relativistic particles $\left(v_{a} \sim 10^{-3} c\right)$, we can reasonably consider the non-relativistic limit of the axion field $\phi_{a}(x, t)$. At first, we separate the rest mass contribution from the time dependence of the axion field as

$$
\phi_{a}(x, t)=\frac{1}{\sqrt{2 m_{a}}}\left(\varphi_{a}(x, t) e^{-i \frac{m_{a} c^{2}}{\hbar} t}+\varphi_{a}^{\dagger}(x, t) e^{i \frac{m_{a} c^{2}}{\hbar} t}\right),
$$

where $\phi_{a}^{\dagger}=\phi_{a}$ is satisfied for the real scalar. Inserting this ansatz into the axion part of the Lagrangian (2.3), we obtain the following non-relativistic action

$$
S_{a}^{\mathrm{NR}}=\int d^{4} x \mathcal{L}_{a}^{\mathrm{NR}}=\int d^{4} x\left[i \hbar \varphi_{a}^{\dagger} \frac{\partial \varphi_{a}}{\partial t}-\frac{\hbar^{2}}{2 m_{a}}\left|\vec{\nabla} \varphi_{a}\right|^{2}\right]
$$

where the second derivative term with respect to time is discarded. This is nothing but the Lagrangian of the Schrödinger field, which satisfies the non-relativistic Schrödinger equation. The non-relativistic axion field $\varphi_{a}(x, t)$ becomes a complex scalar field, whose dimension is given by $\left[L^{-3 / 2}\right]$. Here, we define the rest mass frequency as

$$
\Omega \equiv \frac{m_{a} c^{2}}{\hbar} \simeq 1.5 \times 10^{9}\left[\mathrm{sec}^{-1}\right]
$$

for the typical mass $m_{a} \simeq 10^{-6}\left[\mathrm{eV} / c^{2}\right]$.

In the non-relativistic limit, the axion coupling is also renormalized. Inserting the non-relativistic ansatz of the axion field (2.7) into the original coupling (2.2), we obtain the axion coupling in the non-relativistic limit,

$$
\mathcal{L}_{\mathrm{int}}^{\mathrm{NR}}=g\left(\varphi_{a} e^{-i \Omega t}+\varphi_{a}^{\dagger} e^{i \Omega t}\right) \vec{E} \cdot \vec{B}
$$

Here, the non-relativistic coupling strength is defined as

$$
g \equiv \frac{g_{0}}{\sqrt{2 m_{a}}} \simeq 10^{-29}\left[\frac{\sec C^{2}}{\operatorname{kg} \sqrt{m}}\right],
$$

where the typical value $m_{a} \sim 10^{-6}\left[\mathrm{eV} / c^{2}\right]$ is used. This is the basic formula describing the interaction between the dark matter axions and excitations in condensed matter systems. 


\subsection{Dark matter axion as condensate}

Based on the discussion above, we are lead to a natural but somewhat surprising consequence: the dark matter axions can constitute a (Bose-Einstein (BE)) condensate. At first, the non-relativistic Lagrangian of the axion (2.8) essentially describes non-interacting particles obeying the Bose statistics. ${ }^{3}$ Given the large number density (2.6) and applying the standard argument on the $\mathrm{BE}$ condensation to this axion system, the critical temperature is estimated by $k_{B} T_{c} \simeq\left(2 \pi \hbar^{2} / m_{a}\right) n_{a}^{2 / 3} \sim 10^{7}[\mathrm{eV}]$. Furthermore, the thermal energy of dark matter axions, $k_{B} T_{a}$, is estimated from the average velocity (2.5) to be $k_{B} T_{a} \sim(1 / 2) m_{a} v_{a}^{2} \simeq 10^{-13}[\mathrm{eV}]$. Therefore, if the dark matter axions are in thermal equilibrium in our galaxy, the axion field is naturally expected to be in a BE condensate phase at the extreme low temperature $T_{a}\left(\ll T_{c}\right)$. The possibility of the thermalization of the dark matter axions by the small self-interaction and the gravitational interaction has been recently discussed in the literatures [15-19], and some consequences from the resulting BE condensation have been explored. In this paper, we assume that the dark matter axions do thermalize and form the BE condensate in our galaxy. ${ }^{4}$

Assuming that the dark matter axions are in a condensed phase, we can estimate the occupation number $\mathcal{N}_{k}$ of the mode with the typical momentum $p_{a} \simeq m_{a} v_{a} \sim 10^{-9}[\mathrm{eV} / \mathrm{c}]$. The corresponding thermal de Broglie wave length is given by $\lambda_{a}=2 \pi \hbar / p_{a} \sim 10^{3}[\mathrm{~m}]$, which characterizes the quantum coherence scale of the BE condensation, as discussed in [19]. Using this wave length, the typical occupation number at the mode with $k_{a}=2 \pi / \lambda_{a}$ becomes very large:

$$
\mathcal{N}_{k} \simeq n_{a} \lambda_{a}^{3}=\left(\frac{\rho_{\mathrm{DM}}}{m_{a}}\right) \cdot\left(\frac{2 \pi \hbar}{m_{a} v_{a}}\right)^{3} \simeq 10^{29}
$$

As explained below, this large occupation number $\mathcal{N}_{k}$ gives an enhancement factor for the transition probability, which is analogous to the case of the stimulated emission of photons, used in the LASER. Note that the occupation number $\mathcal{N}_{k}$ is proportional to $m_{a}^{-4}$.

In this condensed regime, the non-relativistic axion field $\varphi_{a}(x, t)$ behaves as a coherent classical field in the leading approximation,

$$
\left\langle\left|\varphi_{a}\right|^{2}\right\rangle \simeq n_{a} \Longrightarrow \varphi_{a} \simeq \varphi_{a}^{\dagger} \simeq \sqrt{n_{a}} \sim 10^{10}\left[\mathrm{~m}^{-3 / 2}\right] .
$$

Using this condensed ansatz of the axion field, we can further rewrite the axion coupling in the following form: ${ }^{5}$

$$
\mathcal{L}_{\text {int }}^{B E}=g\left(\varphi_{a} e^{-i \Omega t}+\varphi_{a}^{\dagger} e^{i \Omega t}\right) \vec{E} \cdot \vec{B} \simeq 2 g \sqrt{n_{a}} \cos (\Omega t) \vec{E} \cdot \vec{B} .
$$

The factor $\sqrt{n_{a}}$ also leads to an enhancement of the coupling strength $g$, and gives a time-periodic coupling for collective excitations, as will be seen in the following.

\footnotetext{
${ }^{3}$ The coupling strength with themselves and other particles are so small that it can be essentially ignored.

${ }^{4}$ It has been argued the possibility that miniclusters composed of axions have been formed through the galaxy formation process $[23,24]$. (For the recent discussions, see $[19,25,26]$.) In this case, we assume that a significant fraction of axions have been thrown off due to the collisions and mergings of such miniclusters, and some fraction of axions forms a smooth halo distribution.

${ }^{5}$ This form of the axion coupling has been frequently discussed in the literature on the detection of dark matter axions, for example [27, 28].
} 


\section{Excitations coupled with axions in condensed matter}

Based on the basic interaction (2.14), three types of excitations in condensed matter systems can possibly couple with the dark matter axions.

(A) Excitations creating the electric field $\vec{E}$ under the external magnetic field $\vec{B}^{\text {ex }}$ :

For concreteness, we consider the homogeneous external magnetic field in the $z$ direction, $\vec{B}^{\mathrm{ex}}=\left(0,0, B_{0}\right)$. In the external magnetic field, the excitations creating the $z$-component of electric field $E_{z}$ can couple with the axions. In this case, the basic coupling (2.14) approximately becomes

$$
\mathcal{L}_{\text {int }}^{B E} \simeq g B_{0}\left(\varphi_{a} e^{-i \Omega t}+\varphi_{a}^{\dagger} e^{i \Omega t}\right)\left(\gamma a+\gamma^{*} a^{\dagger}\right)
$$

Here, the electric field associated with the excitation is given by $E_{z}=\gamma a+\gamma^{*} a^{\dagger}$ in the linear approximation regime, where $a$ is the annihilation operator for a mode of the excitation and $\gamma$ is the corresponding coefficient. The possible candidates for such excitations in condensed matter systems are plasmons in (semi)condcutors, polaritons in insulators, and vortices in superconductors, and so on.

(B) Excitations creating the magnetic field $\vec{B}$ under the external electric field $\vec{E}^{\text {ex }}$ :

Similarly, considering the external electric field in the $z$-direction, $\vec{E}^{\text {ex }}=\left(0,0, E_{0}\right)$, the excitation creating the $z$-component of magnetic field can couple with the axions. Then, the basic coupling (2.14) is given by

$$
\mathcal{L}_{\text {int }}^{B E} \simeq g E_{0}\left(\varphi_{a} e^{-i \Omega t}+\varphi_{a}^{\dagger} e^{i \Omega t}\right)\left(\gamma a+\gamma^{*} a^{\dagger}\right)
$$

where the magnetic field associated with the excitation is assumed to be $B_{z}=\gamma a+$ $\gamma^{*} a^{\dagger}$. The candidates are magnons and domain walls in magnetic systems, etc.

(C) Excitations which can create both the electric and magnetic field:

Recent studies on multiferroic and topological materials show that there exist the excitations creating both the electric field in response to an external magnetic field and the magnetic field in response to an external electric field. Through the magnetoelectric effect, these excitations have the coupling with the axions in the form of (3.1) or (3.2), depending on the external field. The interesting candidates for such excitations are skyrmions in chiral-lattice magnets [29] and condensed matter analogues of the axions in a topological insulator with time-reversal breaking (so-called topological magnetic insulator) [30, 31]. ${ }^{6}$

In the following, we focus on the case of the excitations of type A, since the discussions are almost parallel for those of type B and C. In particular, as a concrete example and an interesting possibility, the magnetic vortex strings in type II superconductors will be discussed thoroughly.

\footnotetext{
${ }^{6} \mathrm{~A}$ realization of axion electrodynamics is also discussed by using optical lattices [32].
} 


\section{Axion-vortex coupling in type II superconductors}

In this section, we discuss the effects of the axion coupling (2.10) in the dynamics of type II superconductors and, in particular, magnetic vortex strings.

\subsection{Ginzburg-Landau description of superconductor and magnetic vortex}

We start with the Ginzburg-Landau (GL) equations, which give the effective description of type II superconductors [33, 34].

$$
\begin{aligned}
0 & =\frac{\hbar^{2}}{2 m^{*}}\left(\vec{\nabla}-i \frac{e^{*}}{\hbar} \vec{A}\right)^{2} \psi-\alpha \psi-\beta|\psi|^{2} \psi, \\
\frac{1}{\mu_{0}}(\vec{\nabla} \times \vec{B}) & =i \frac{\hbar e^{*}}{2 m^{*}}\left[\left(\vec{\nabla} \psi^{\dagger}\right) \psi-\psi^{\dagger}(\vec{\nabla} \psi)\right]-\frac{e^{* 2}}{m^{*}}|\psi|^{2} \vec{A} .
\end{aligned}
$$

Here, we denote the order parameter of superconductors as $\psi$, and $e^{*}$ and $m^{*}$ are the electric charge and mass of the order parameter, respectively. $\alpha$ and $\beta$ correspond to the parameters which can be calculated from the microscopic theory à la BCS.

From the second equation (4.2), we can read the supercurrent,

$$
\begin{aligned}
\vec{J}_{s} & =i \frac{\hbar e^{*}}{2 m^{*}}\left\{\left(\vec{\nabla} \psi^{\dagger}\right) \psi-\psi^{\dagger}(\vec{\nabla} \psi)\right\}-\frac{e^{* 2}}{m^{*}}|\psi|^{2} \vec{A} \\
& =\frac{\hbar e^{*}}{m^{*}}|f|^{2}\left(\vec{\nabla} \theta-\frac{e^{*}}{\hbar} \vec{A}\right),
\end{aligned}
$$

where we insert the decomposition of the order parameter, $\psi(x)=f(x) e^{i \theta(x)}$, for the second equality.

As is well-known, the GL equations have the magnetic vortex solutions that are topologically stable $[35,36]$. For the later discussion, we briefly summarize the solitonic vortex solution. A straight single vortex, which is stretched in the $z$-direction, is described by the field configuration with the cylindrical symmetry:

$$
\vec{A}_{\mathrm{v}}(x)=\left(\frac{\hbar}{e^{*}}\right)\left(\frac{\vec{d}_{z} \times \vec{r}}{r}\right) A(r), \quad \psi_{\mathrm{v}}(x)=\sqrt{\frac{|\alpha|}{\beta}} e^{i \theta} f(r) .
$$

Here, $(r, \theta, z)$ are the cylindrical coordinates, and $\vec{d}_{z}$ is the unit vector in the $z$-direction. $A(r)$ and $f(r)$ are the functions of the radial coordinate $r$ only, and the flux is normalized to be the minimal flux quantum, $h / e^{*}$. With these ansatze, the GL equations are reduced to the following coupled ordinary differential equations with respect to $r$ :

$$
\begin{aligned}
& 0=\xi^{2}\left[\frac{1}{r} \frac{d}{d r}\left(r \frac{d f(r)}{d r}\right)-\left(\frac{1}{r}-A(r)^{2}\right) f(r)\right]-f(r)+f(r)^{3}, \\
& 0=\frac{d}{d r}\left[\frac{1}{r} \frac{d}{d r}(r A(r))\right]-\frac{\mu_{0} e^{* 2}|\alpha|}{m^{*} \beta}\left(A(r)-\frac{1}{r}\right) f(r)^{2} .
\end{aligned}
$$

Here, we define the coherence length for the order parameter:

$$
\xi=\sqrt{\frac{\hbar^{2}}{2 m^{*}|\alpha|}} .
$$


With the boundary conditions $f(r) \rightarrow 1$ and $A(r) \rightarrow 1 / r$ at $r \rightarrow \infty,{ }^{7}$ we can easily obtain the numerical solutions for these equations, which describe the straight vortex with minimal flux. Away from the vortex core $(f(r) \simeq 1)$, we can obtain another characteristic length scale,

$$
\lambda_{L}=\sqrt{\frac{m^{*} \beta}{\mu_{0} e^{* 2}|\alpha|}},
$$

which defines the penetration depth of the electromagnetic field in superconductors.

\subsection{Vortex dynamics with axion coupling}

Now, we consider the interaction between the axion fields $\varphi_{a}$ and magnetic vortex strings based on the axion coupling (2.10). Since the vortex string is a typical example of the excitations of type A, we consider the external magnetic field $\vec{B}^{\text {ex }}$ in the $z$-direction, $B_{z}^{\text {ex }}=$ $B_{0}=$ const. $\left(B_{x}^{\text {ex }}=B_{y}^{\text {ex }}=0\right)$. Then, the axion coupling becomes

$$
\mathcal{L}_{\text {int }}^{\mathrm{NR}} \simeq g B_{0}\left(\varphi_{a} e^{-i \Omega t}+\varphi_{a}^{\dagger} e^{i \Omega t}\right) E_{z}
$$

Here, we derive the electric field created by the vortex motion in a simple set-up. For concreteness, we consider the limit of large penetration depth and small coherence length, compared to the size of a superconducting sample. ${ }^{8}$ In this situation, an external magnetic field becomes almost homogeneous in the sample and the core of magnetic vortex becomes infinitely thin. ${ }^{9}$ Outside the core of vortex, the GL equation (4.2) and supercurrent (4.3) give $\vec{A}=\left(\hbar / e^{*}\right) \vec{\nabla} \theta$. From the AC Josephson effect and gauge invariance, an electrostatic potential is also given by $\Phi=-\left(\hbar / e^{*}\right) \dot{\theta}$. Since the motion of the vortex with (small) velocity $\vec{v}_{L}$ implies a time-dependent phase $\theta=\theta\left(\vec{x}-\vec{v}_{L} t\right)$, the electric field originated from the vortex motion is obtained as

$$
\begin{aligned}
\vec{E}_{\mathrm{vor}}=-\vec{\nabla} \Phi-\frac{\partial \vec{A}}{\partial t} & =\left(\frac{\hbar}{e^{*}}\right)\left(\vec{\nabla} \frac{\partial}{\partial t}-\frac{\partial}{\partial t} \vec{\nabla}\right) \theta\left(\vec{x}-\vec{v}_{L} t\right) \\
& =-\left(\frac{h}{e^{*}}\right)\left(\vec{v}_{L} \times \vec{d}\right) \delta^{2}(\vec{x}),
\end{aligned}
$$

where the multivalued property of the phase $\theta(x)$ is used, and $\vec{d}$ is a unit vector along the vortex axis, and the two-dimensional delta function has the support on the core of vortex.

As seen from the vortex electric field (4.10), the motion in the plane perpendicular to the vortex axis is important, and we focus on the perpendicular motion in the following. Concretely, we regard the vortex string as a point particle on the perpendicular plane with a mass $m_{\text {vor }}$, by integrating out the spatial coordinate dependence in the Lagrangian. Using the formula (4.10) and the homogeneous property (2.13) of $\varphi_{a}$, the axion coupling

\footnotetext{
${ }^{7}$ For non-singular solutions, the boundary condition $f(r) \rightarrow 0$ at $r \rightarrow 0$ is also imposed.

${ }^{8}$ Some compound superconductors, such as $\mathrm{NbN}$, have the coherence length $\xi \sim 1 \mathrm{~nm}$ and penetration depth $\lambda_{L} \sim 1 \mu \mathrm{m}$, and the samples of the size $\sim 100 \mathrm{~nm}$ can be fabricated.

${ }^{9}$ In the small $\xi$ limit, we take the order parameter as $\psi(x)=f e^{i \theta(x)}$ with a constant $f$.
} 
can be written in the minimal coupling form,

$$
L_{\mathrm{int}}=\int d^{3} x \mathcal{L}_{\mathrm{int}}^{\mathrm{NR}} \simeq\left(\frac{g h \ell_{\mathrm{vor}}}{e^{*}}\right)\left(\varphi_{a} e^{-i \Omega t}+\varphi_{a}^{\dagger} e^{i \Omega t}\right)\left(\vec{B}^{\mathrm{ex}} \times \vec{d}\right) \cdot \vec{v}_{L}=\overrightarrow{\mathcal{A}}_{\mathrm{eff}} \cdot \vec{v}_{L},
$$

where $\ell_{\text {vor }}$ is the length of the vortex string. Here, the effective gauge field $\overrightarrow{\mathcal{A}}_{\text {eff }}$ is introduced and its magnitude is given by

$$
\left|\mathcal{A}_{\mathrm{eff}}\right| \simeq\left(\frac{g h\left|\varphi_{a}\right| B_{0} \ell_{\mathrm{vor}}}{e^{*}}\right)
$$

The above analysis can be simply extended to the case of general type II superconductors, using the time-dependent Ginzburg-Landau theory [34, 37].

Note that, for this coupling to work, we require the existence of the vortices or segments of the vortex whose axes are non-parallel to the external magnetic field $\vec{B}^{\text {ex }}$, as is evident from (4.11). For this purpose, we consider the following types of configurations of the vortex strings: ${ }^{10}$

(a) Tilted vortex strings against a tuned external magnetic field, due to a boundary condition of a small superconducting sample.

(b) Wavy vortex strings with the non-paralell segments or the vortex strings with the kink-like segments, due to the small tension near the phase transition.

In the following, we discuss mainly the case of the tilted vortex strings of type (a). For the wavy or kink-like vortex strings, the calculations can be done in the same way, except for replacing the length and number of the vortex strings with those of the segments.

It should be noted the more physical situation behind the above discussion. We consider a vortex ensemble, which is composed of many vortices, in a type II superconductor, and assume that each vortex can move in an independent way in the ensemble. Such an ensemble can be realized in a vortex liquid phase near the superconducting critical point. When the number of vortices and the penetration depth are large, the magnetic field of each vortex is overlapped each other, and the averaged magnetic field becomes approximately homogeneous in a small sample. In such a situation, the dynamics of a single vortex can be described as the motion of a vortex core, where the order parameter vanishes, in the background (averaged) magnetic field $\vec{B}^{\text {ex }}$. Using this physical picture, the same result can be obtained also in the rest frame of the moving vortex. In this frame, the vortex is at rest, and the electric field created by the vortex is vanishing. However, the averaged magnetic field (or other vortices) are moving with the velocity $-\vec{v}_{L}$, and the Galilei transformation (or the small velocity limit of the Lorentz boost) of the magnetic field $\vec{B}^{\text {ex }}$ leads to an electric field $\vec{E}=\vec{v}_{L} \times \vec{B}^{\mathrm{ex}}[40]$. This electric field and the quantized magnetic flux $(\propto \vec{d})$ of the vortex reproduce the same coupling (4.11).

\footnotetext{
${ }^{10}$ In numerical simulations of type II superconductors, various non-parallel vortex strings to an external magnetic field have been recognized, due to the boundary conditions [38] and the pinning effects [39].
} 


\subsection{Stimulated emission of axions from vortices}

The axion coupling (4.11) and a standard kinetic term lead to the effective Lagrangian of the vortex string dynamics,

$$
L_{p}=\frac{m_{\mathrm{vor}}}{2} \vec{v}_{L}^{2}+\overrightarrow{\mathcal{A}}_{\mathrm{eff}}(t) \cdot \vec{v}_{L}
$$

where $\vec{v}_{L}$ is the velocity of the center-of-mass of the vortex on the perpendicular plane. Thus, our problem on the perpendicular motion of a rigid vortex with the axion coupling (4.9) is reduced to the problem of a charged particle coupled with an "effective" AC electric field, whose frequency is $\Omega \sim 1[\mathrm{GHz}]$ and magnitude is given by

$$
\left|\overrightarrow{\mathcal{E}}_{\mathrm{eff}}\right| \simeq\left(\frac{g h \Omega \sqrt{n_{a}} B_{0} \ell_{\mathrm{vor}}}{e^{*}}\right)
$$

where we have used the ansatz of the BE condensation of the axion field (2.13).

In this section, using the effective Lagrangian, we estimate the emission probability of axions from a vortex motion, within the first order perturbation theory, i.e. the Fermi's golden rule. In order to realize the stimulated emission process by using the coupling (4.11), which is originated from the axion coupling (2.2), we require that there exist the excited states of the vortex dynamics having the energy level $\Delta E \sim \hbar \Omega$, which enables the emission of the non-relativistic axions with the energy, $E_{a} \simeq m_{a} c^{2}+\frac{m_{a}}{2} v_{a}^{2}$. Furthermore, as in the case of the LASER, the non-equilibrium situation between the vortex system and the axions is also required, and the enough number of states of the vortices should be excited from the ground state. This implies that the vortex system should have a higher temperature than both the axion temperature $T_{a}$ and $T_{\text {gap }}=\Delta E / k_{B}$. Therefore, we make the following assumptions on the dynamics of vortices in type II superconductors:

(I) The energy spectrum of the vortex dynamics has the excitations of the order of $\hbar \Omega \sim 10^{-6} \mathrm{eV}$, which corresponds to the energy of the condensed axions.

(II) The vortices in the superconductor are in thermal equilibrium of the temperature $T_{S C}$, higher than $T_{a}$ and $T_{\text {gap }}$, without considering the effect of the dark matter axions.

To realize the assumption (I), we consider the magnetic vortices in mobile vortex systems where the excitaions with such a small energy level can be realized and observed as a resonance in experiments with microwave around GHz. Such mobile vortices can be realized in vortex creep, vortex flow, and vortex liquid in a type of superconductors [41]. For the assumption (II), we take the typical temperature of a mobile vortex ensemble in superconductors as $T_{S C} \sim 4 K,{ }^{11}$ where the thermal energy of the vortex is typically $k_{B} T_{S C} \simeq 10^{-4} \mathrm{eV}$. It should be noted that the energy spectrum of the vortex dynamics in mobile vortex systems is expected to be broad around $\Delta E$, and the emission can take place within a certain range of the axion mass.

\footnotetext{
${ }^{11}$ The experiments on superconductors are often performed at the liquid Helium temperature, $4.2 \mathrm{~K}$.
} 
In the second quantized language, the non-relativistic axion fields $\varphi_{a}$ has the Fourier expansion,

$$
\begin{aligned}
\varphi_{a}(x, t) & =\sum_{k} \frac{1}{\sqrt{V_{a}}} a_{k} e^{i \vec{k} \cdot \vec{x}-i \omega t}, \\
{\left[a_{k}, a_{k^{\prime}}^{\dagger}\right] } & =\delta_{k, k^{\prime}}, \quad\left[a_{k}, a_{k^{\prime}}\right]=\left[a_{k}^{\dagger}, a_{k^{\prime}}^{\dagger}\right]=0 .
\end{aligned}
$$

Here, $V_{a}$ is the volume of a box where the axion field is defined, and we finally take the limit $V_{a} \rightarrow \infty$ (or cosmological scale) as usual. Inserting the Fourier expansion (4.15), the axion coupling becomes

$$
\begin{aligned}
\int d^{3} x g\left(\varphi_{a} e^{-i \Omega t}+\varphi_{a}^{\dagger} e^{i \Omega t}\right) \vec{E} \cdot \vec{B} & \simeq\left(\frac{g h B_{0} \ell_{\mathrm{vor}}}{e^{*} \sqrt{V_{a}}}\right) \sum_{k \simeq k_{a}}\left(a_{k} e^{-i \Omega t}+a_{k}^{\dagger} e^{i \Omega t}\right) \vec{n}_{A} \cdot \vec{v}_{L} \\
& \equiv \overrightarrow{\mathcal{A}}_{\mathrm{eff}}(t) \cdot \vec{v}_{L}
\end{aligned}
$$

where $\vec{n}_{A}=\left(\vec{B}^{\mathrm{ex}} \times \vec{d}\right) /\left|B^{\mathrm{ex}}\right|$ is a unit vector in the direction of the effective gauge field. Here, we used the low-energy approximation, $e^{i \vec{k} \cdot \vec{x}} \sim 1$ and $\Omega \gg \omega$, and the axion distribution, which is highly concentrated around $k \simeq k_{a}$ based on the assumption of the axion condensation.

Since this coupling is the same form as the standard minimal coupling to the EMfield, the interaction Hamiltonian, which contributes the perturbative calculation, simply becomes

$$
H_{\text {int }}(t)=-\frac{\overrightarrow{\mathcal{A}}_{\text {eff }}(t) \cdot \vec{p}_{L}}{m_{\text {vor }}}
$$

From this interaction Hamiltonian, we can estimate the emission probability within the first-order perturbation, by using the Fermi's golden formula,

$$
\text { Prob. }\left[\sec ^{-1}\right]=\int \frac{d^{3} k V_{a}}{(2 \pi)^{3}}\left(\frac{2 \pi}{\hbar}\right)\left|\left\langle f\left|H_{\mathrm{int}}(\Omega)\right| i\right\rangle\right|^{2} \delta\left(E_{i}-E_{f} \pm \hbar \Omega\right),
$$

where the momentum integration is performed over the emitted axion in the final state. Since we assume that the temperature of the vortex system $\left(T_{S C} \sim 4 K\right)$ is much higher than the temperature of the condensed axions $\left(T_{a} \sim 10^{-9} \mathrm{~K}\right)$, the axion emission process is dominant. Furthermore, due to the assumption that the axions form the BE condensate in our background, the emission probability is largely enhanced by the huge occupation number $\mathcal{N}_{k} \sim 10^{29}$ for the emission process of the non-relativistic axion into the condensate, and such a process becomes dominant, as in the case of LASER. Therefore, we specifically consider the process with the initial state $|i\rangle=\left|E_{1}\right\rangle_{v} \otimes\left|\mathcal{N}_{k}\right\rangle_{a}$, and the final state $|f\rangle=$ $\left|E_{0}\right\rangle_{v} \otimes\left|\mathcal{N}_{k}+1\right\rangle_{a}$, where $\Delta E=E_{1}-E_{0} \simeq m_{a} c^{2}$. Here, $\left|E_{i}\right\rangle_{v}$ is the states of the vortex with the energy $E_{i}$, and $\left|\mathcal{N}_{k}\right\rangle_{a}$ is the condensed state of the axions with the occupation number $\mathcal{N}_{k}$ of the condensed mode $k_{a}$. (See also the figure 1.) Although there exist higher excited states of the vortex dynamics, the emission probability of relativistic axions from such states with the typical energy $E_{a} \sim k_{B} T_{S C}$ is negligibly small, beacuse of the absence of the enhancement factor, and such processes can be essentially ignored. 


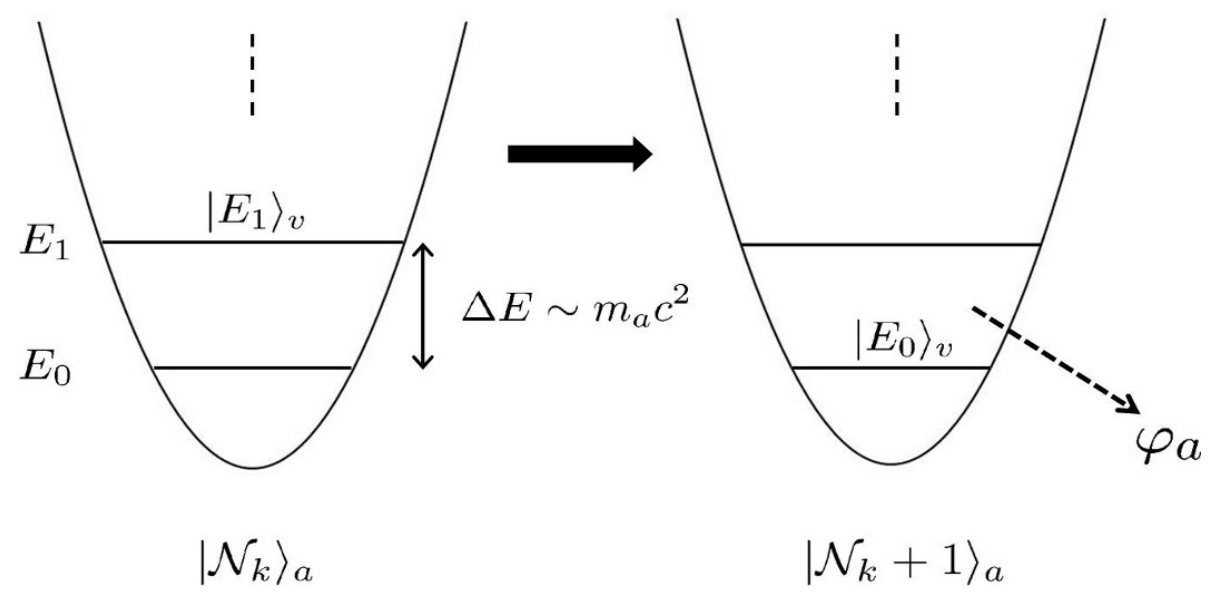

Figure 1. The schematic picture of the stimulated axion emission from the vortex excitation.

In the condensed phase, the state can be expressed by a coherent state, which is an eigenstate of the annihilation operator:

$$
a_{k}\left|\mathcal{N}_{k}\right\rangle \propto \sqrt{\mathcal{N}_{k}}\left|\mathcal{N}_{k}\right\rangle, \quad\left\langle\mathcal{N}_{k} \mid \mathcal{N}_{k}\right\rangle=1
$$

From this property, the matrix element for the axion sector is evaluated as

$$
\left\langle f\left|a_{k}^{\dagger}\right| i\right\rangle={ }_{a}\left\langle\mathcal{N}_{k}+1\left|a_{k}^{\dagger}\right| \mathcal{N}_{k}\right\rangle_{a} \simeq \sqrt{\mathcal{N}_{k}}
$$

Inserting the explicit form of $\overrightarrow{\mathcal{A}}_{\text {eff }}(t)$ into the formula (4.19) and taking the emission part proportional to $\delta\left(E_{i}-E_{f}-\hbar \Omega\right)$, the probability becomes

$$
\text { Prob. }\left[\mathrm{sec}^{-1}\right]=\left(\frac{g B_{0} \ell_{\mathrm{vor}}}{e^{*} m_{\mathrm{vor}}}\right)^{2} \hbar \mathcal{N}_{k} \int d^{3} k\left|\left\langle p_{L}\right\rangle\right|^{2} \delta\left(E_{i}-E_{f}-\hbar \Omega\right),
$$

where the matrix element for the vortex sector is denoted as $\left\langle p_{L}\right\rangle \equiv{ }_{v}\left\langle E_{0}\left|p_{L}\right| E_{1}\right\rangle_{v}$. Note that the (apparent) dependence of the volume $V_{a}$ is cancelled in the resulting probability formula.

Further assuming the matrix element $\left\langle p_{L}\right\rangle$ is independent of the momentum of the emitted axion in the low-energy region, we can perform the momentum integration,

$$
\int d^{3} k \delta\left(E_{i}-E_{f}-\hbar \Omega\right)=4 \sqrt{2} \pi \frac{m_{a}^{3 / 2} \sqrt{\hbar \Omega}}{\hbar^{3}} .
$$

Gathering the above formulas, we have the expression for the emission probability of the axion from a single vortex,

$$
\text { Prob. }\left[\sec ^{-1}\right]=\mathcal{N}_{k}\left(4 \sqrt{2} \pi m_{a}^{3 / 2} \sqrt{\hbar \Omega}\right)\left(\frac{g B_{0}}{\hbar e^{*} \rho_{\mathrm{vor}}}\right)^{2}\left|\left\langle p_{L}\right\rangle\right|^{2},
$$

where we defined the vortex mass density $\rho_{\mathrm{vor}}=m_{\mathrm{vor}} / \ell_{\mathrm{vor}}[\mathrm{kg} / m]$. 
Finally, in order to evaluate the matrix element $\left|\left\langle p_{L}\right\rangle\right|^{2}$, we consider a simple model of the vortex dynamics, for concreteness. The model is described by the Hamiltonian with an effective harmonic potential [41]: ${ }^{12}$

$$
H_{\mathrm{vor}}=\frac{p_{L}^{2}}{2 m_{\mathrm{vor}}}+\frac{m_{\mathrm{vor}} \Omega^{2}}{2} x^{2}=\hbar \Omega a^{\dagger} a,
$$

where the creation and annihilation operators, $\left[a, a^{\dagger}\right]=1$, are introduced. According to our assumption (I), the energy level should be $\hbar \Omega \simeq m_{a} c^{2} \sim 10^{-6}[\mathrm{eV}]$. Using the relation $p_{L}=\sqrt{\frac{m_{\mathrm{vor}} \hbar \Omega}{2}}\left(a+a^{\dagger}\right)$, the matrix element can be estimated as $\left|\left\langle p_{L}\right\rangle\right|^{2} \sim m_{\mathrm{vor}}(\hbar \Omega)$. With this estimation, the emission probability from a single vortex (or a single segment of the vortex) becomes

$$
\text { Prob. }\left[\mathrm{sec}^{-1}\right] \simeq \mathcal{N}_{k}\left(4 \sqrt{2} \pi m_{\mathrm{vor}}\left(m_{a} \hbar \Omega\right)^{3 / 2}\right)\left(\frac{g B_{0}}{\hbar e^{*} \rho_{\mathrm{vor}}}\right)^{2} .
$$

If an experimental sample of a superconductor contains the multiple vortices, the probability is proportional to the number of the vortices (for the tilted vortices) or the number of segments (for the wavy or kink-like vortices), which are denoted as $N_{\text {vor }}$, and the fraction factor $f\left(E_{1}\right)$, which is the fraction of the vortex states with the energy level $E_{1} \cdot{ }^{13}$ Thus, the emission probability of the dark matter axions from the vortex ensemble in type II superconductors becomes

$$
\text { Prob. }\left[\sec ^{-1}\right] \simeq \mathcal{N}_{k} N_{\text {vor }} f\left(E_{1}\right)\left(4 \sqrt{2} \pi m_{\text {vor }}\left(m_{a} \hbar \Omega\right)^{3 / 2}\right)\left(\frac{g B_{0}}{\hbar e^{*} \rho_{\text {vor }}}\right)^{2} .
$$

As the typical values of an experimental set-up, using $B_{0} \sim 10[\mathrm{~T}], \rho_{\mathrm{vor}} \sim 10^{6}\left[\mathrm{eV} / \mathrm{c}^{2} \mathrm{~m}\right]$, $\ell_{\text {vor }} \sim 10^{-4}[\mathrm{~m}]$, and $N_{\text {vor }} \sim 10^{6}$ with $f\left(E_{1}\right) \sim 10^{-2}$, we can numerically estimate the probability,

$$
\text { Prob. } \simeq 10^{9}\left[\mathrm{sec}^{-1}\right]
$$

Our result implies that, in an appropriate circumstances, the emission probability of the dark matter axions can be sizable, even if the axion coupling strength $g_{0}$ is quite small. For this type of process, the Bose statistics and small mass of the dark matter axions are crucial, which distinguishes from other dark matter candidates, such as WIMPs. We comment here the difference from the conventional conversion process of axions into photons with a background magnetic field through the axion coupling. In this process, a mode mixing between the coherent axion field and the EM-field is essential, where the both fields are treated as classical waves, and the aforementioned stimulated emission does not play any role $[45,46]$. In our axion emission process, the vortex is a heavy localized object compared to the axion, and thus the spontaneous emission of axions can be discussed in analogy with the case of spontaneous radiation from an atom.

\footnotetext{
${ }^{12}$ Another model can be considered based on the transverse oscillations of the vortex string with a small tension.

${ }^{13}$ While this fraction factor can be calculated from the statistical mechanics of the vortices in principle, it generally depends on the details of the vortex dynamics.
} 


\section{Possible signature of dark matter axions}

An immediate consequence from the stimulated emission of dark matter axions is the axiondriven non-equilibrium states of the vortex ensemble in type II superconductors [33, 34]. Emitted axions from the vortices are almost invisible, because the interactions with other particles are negligibly small. However, the vortices consequently lose thermal energy due to the emission of axions, by the amount, $E_{\text {lost }} \simeq$ Prob. $\times m_{a} c^{2}[\mathrm{eV} / \mathrm{sec}]$. This process lowers the (local) temperature of the vortex system, and leads to the non-equilibrium state in the vortex ensemble. It should be emphasized that the temperature of the condensate of the dark matter axions is extremely low, $T_{a} \sim 10^{-9} \mathrm{~K}$, and the superconductor with vortices has the higher temperature $T_{\mathrm{SC}} \sim \mathcal{O}(1) K$. Thus, the coupled system of the axions and vortices cannot reach the equilibrium as a whole in the laboratory. One possible experimental signature of the axion-driven non-equilibrium can be detected by the temperature measurement of a superconducting sample with mobile vortices in the vortex flow or vortex liquid. If the stimulated emission discussed in the last section is indeed realized, the adiabatic superconducting sample lowers its temperature spontaneously, whereas its temperature normally should rise, due to the interaction with environments, in the absence of such an emission.

Another possibility is to detect the transport phenomena of the magnetic vortices in type II superconductors, such as the vortex Hall effect and vortex Nernst effect from the vortex motions $[33,34,41]$, in a non-equilibrium situation due to the axion condensate. Note that the energy loss of vortices due to frictions from phonons or other excitations in superconductors can be estimated by standard arguments in statistical physics such as the fluctuation-dissipation theorem, and can be distinguished from the axion effect.

Indeed, the axion emission in the vortex ensemble locally causes the temperature gradients, and leads to an extra random noise in the vortex motion. The noise originating from the vortex motion in the vortex liquid has been detected up to the smallest magnitude $10^{6}[\mathrm{eV} / \mathrm{sec}][42,43]$. Our estimation implies the magnitude of the axion-induced noise is $10^{3}[\mathrm{eV} / \mathrm{sec}]$, and, if it exists, this noise can be detected in the current or future experiments. Furthermore, the numerical simulation of the vortex Nernst effect, which originates from the vortex motion due to thermal gradients, has been developed [44] using the time-dependent Ginzburg-Landau theory with a thermal noise, and the comparison between the results of experiments and simulations can reveal the axion emission effect in the moblie vortex system.

\subsection{Stimulated emission from other excitations in condensed matter}

So far, we have intensively discussed the stimulated emission from the magnetic vortices in superconductors, and estimated the emission rates, as a concrete example. As discussed in section 3 , there are other types of excitations which can couple with the dark matter axions via the coupling (2.14). If such excitations have the energy level $\Delta E \simeq \hbar \Omega \sim 10^{-6}[\mathrm{eV}]$, the same arguments as the vortex case can be applied straightforwardly.

At first, we consider an excitation in type A with the external magnetic field $\vec{B}^{\text {ex }}=$ $\left(0,0, B_{0}\right)$. In the linear approximation, the electric field created by such an excitation 
is represented by $E_{z}=\gamma a+\gamma^{*} a^{\dagger}$, with the annihilation and creation operators of the excitation mode. ${ }^{14}$ In this case, the stimulated emission rate is generally given by the following formula

$$
\text { Prob. }\left[\sec ^{-1}\right]=\mathcal{N}_{k} N_{\mathrm{exc}}\left(4 \sqrt{2} \pi m_{a}^{3 / 2} \sqrt{\hbar \Omega}\right)\left(\frac{g B_{0}|\gamma|}{2 \pi \hbar}\right)^{2} .
$$

Here, the number of the excitations in the sample is given by $N_{\text {exc }}$, and the excitation level is assumed to be $\hbar \Omega \simeq m_{a} c^{2}$. For the excitations of type $\mathrm{B}$, which create a magnetic field under the background electric field $\vec{E}^{\text {ext }}=\left(0,0, E_{0}\right)$, similar calculations based on the coupling (3.2) can be applied, and we can obtain the emission rate,

$$
\text { Prob. }\left[\mathrm{sec}^{-1}\right]=\mathcal{N}_{k} N_{\text {exc }}\left(4 \sqrt{2} \pi m_{a}^{3 / 2} \sqrt{\hbar \Omega}\right)\left(\frac{g E_{0}|\gamma|}{2 \pi \hbar}\right)^{2},
$$

where $N_{\text {exc }}$ is the number of the excitations with $\Delta E \simeq \hbar \Omega$ in the sample.

If the excitations of type $\mathrm{C}$ in a multiferroic (or topological) material also have the energy spectrum of the order $10^{-6}[\mathrm{eV}]$, the stimulated emission of axions can be realized with either an external electric field or a magnetic field via the coupling (3.1) or (3.2). The emission rate is given by the formulas (5.1) or (5.2) in the same way.

\section{Summary and discussion}

We have discussed the coupling between dark matter axions and excitations in various condensed matter systems, and physical consequences from the coupling. In particular, we investigated the stimulated emission of dark matter axions, which are expected to take place $\mathrm{BE}$ condensates in our galaxy, from the various excitations in condensed matter systems through the axion coupling. As a concrete example, the emission from magnetic vortex strings of mobile vortex systems in superconductors was investigated, and a possible experimental signature, that is the spontaneous cooling and the resulting non-equilibrium state of the vortex ensemble, was discussed. It is expected that the stimulated emissions possibly give a new strategy to detect dark matter axions. For a more concrete analysis to detect the experimental signals, further understanding of dynamics of the vortex strings in the mobile vortex systems, such as vortex flow and vortex liquid states, is required, and the investigation of such vortex dynamics is a work in progress. Although we focused on the vortex strings in s-wave superconductors in this paper, the axion coupling and emission in the case of vortex strings in (chiral) p-wave supercondutors should also be interesting.

Generalizations to other dark matter models with axion-like particles are straightforward. The emission rates are proportional to $g_{0}^{2} / m_{a}^{2}$, where $g_{0}$ and $m_{a}$ are the coupling strength and mass of the dark matter axion, and are enhanced for smaller mass axion if condensed matter excitations have the energy spectrum $\hbar \Omega \sim m_{a} c^{2} .{ }^{15}$

\footnotetext{
${ }^{14}$ For the vortex case, $\gamma=\gamma^{*}=\left(\frac{h}{e^{*} \rho_{\mathrm{vor}}}\right) \sqrt{\frac{m_{\mathrm{vor}} \hbar \Omega}{2}}$.

${ }^{15}$ Recently, new detection methods of ultralight axion-like particles corresponding to BEC dark matters are proposed. See [28, 47, 48] for example.
} 
There is another interesting problem: the excitation of type $\mathrm{C}$, such as an analogue of the axion in a topological magnetic insulator, has the same effective coupling as the axion coupling. Thus the mixing between the dark matter axions and condensed matter axions can occur in principle. Physical consequences from such a mixing will be discussed in a future work.

From the perspective of thermodynamics, the axion condensate plays the role of heat bath at an extremely low temperature, for the condensed matter excitations discussed in this paper. Therefore, the mechanism investigated in this paper can lead to a novel energy conversion process from the condensed matter excitations.

\section{Acknowledgments}

The authors thank J. Lustikova, S. Murakami, K. Sato, Y. Shiomi, and F. Takahashi for useful discussions. This work was supported in part by Grant-in Aid for Scientific Research on Innovative Areas "Nano Spin Conversion Science" (26103005). The work of E. S. was supported in part by JST ERATO Grant Number JPMJER1402, Japan.

\section{A Dimensional analysis on axion coupling}

In this appendix, we determine the unit (or dimension) of the coupling strength $g_{0}$ between the axion and EM-field. In the following, we denote the dimension of the Mass, Length, and Time as $[M],[L]$, and $[T]$, respectively. For the units of the EM-field, we take the electric charge as the fourth element of our unit, and denote the dimension of charge as $[Q]$. Here, we take the MKSC units (C represents [Coulomb]).

Since the dimension of the action $S$ is given by $[S]=\left[M L^{2} T^{-1}\right]$, we can determine the dimension of $\phi_{a}$,

$$
\left[\phi_{a}\right]=\left[M^{-1 / 2} L^{-3 / 2}\right] .
$$

As is well-known, the dimensions of the EM-field are determined from the equations of motion for the charged particles,

$$
[\vec{E}]=\left[M L T^{-2} Q^{-1}\right], \quad[\vec{B}]=\left[M T^{-1} Q^{-1}\right] .
$$

From these relations, we obtain the dimension of the vector potential, $[\vec{A}]=\left[M L T^{-1} Q^{-1}\right]$.

From the above dimensions, we have the dimension of the following term (operator),

$$
\left[\phi_{a} \vec{E} \cdot \vec{B}\right]=\left[M^{3 / 2} L^{-1 / 2} T^{-3} Q^{-2}\right] .
$$

Using the $[S]=\left[M L^{2} T^{-1}\right]$, we can determine the dimension of the axion coupling strength,

$$
\left[g_{0}\right]=\left[M^{-1 / 2} L^{-1 / 2} T Q^{2}\right] .
$$

Thus, the coupling strength $g_{\mathrm{mks}}$ in the MKSC unit becomes

$$
g_{0}=g_{\mathrm{mks}}\left[\frac{\sec C^{2}}{\sqrt{\mathrm{kg} m}}\right] \text {. }
$$


The experimental bound (or constraint) of the value of the axion coupling strength is usually discussed in the natural units over the communities of high-energy physics, cosmology and astrophysics. To convert the MKSC units into the natural units, the following conversion formulas are useful:

$$
\begin{aligned}
1 \mathrm{~kg} & =5.61 \times 10^{35}\left[\mathrm{eV} / \mathrm{c}^{2}\right], \\
1 m & =5.07 \times 10^{6}[\hbar c / \mathrm{eV}], \\
1 \mathrm{sec} & =1.52 \times 10^{15}[\hbar / \mathrm{eV}], \\
1 \mathrm{eV} & =1.60 \times 10^{-19}[\mathrm{Joule}],
\end{aligned}
$$

where $c=3.00 \times 10^{8} \mathrm{~m} / \mathrm{sec}$ is the light velocity.

In the natural units, we set $c=\hbar=\varepsilon_{0}=\mu_{0}=1$. The (dimensionless) fine structure constant $\alpha=e^{2} / 4 \pi \varepsilon_{0} \hbar c=7.30 \times 10^{-3}(\sim 1 / 137)$ gives the elementary electric charge,

$$
e=\left(4 \pi \alpha \varepsilon_{0} \hbar c\right)^{1 / 2}[C]
$$

and $e=0.303$ (dimensionless) in the natural units.

Thus, the axion coupling strength in the natural units $g_{\text {nat }}$ is given by

$$
g_{\mathrm{nat}} \simeq 3.22 \times 10^{30} \times g_{\mathrm{mks}}\left[\mathrm{eV}^{-1}\right] .
$$

The upper bounds of this coupling strength originate from various astronomical observations of sun, neutron stars, and supernovae, which lead to

$$
g_{\text {nat }} \lesssim 10^{-19}\left[\mathrm{eV}^{-1}\right] \quad \Longrightarrow \quad g_{\mathrm{mks}} \lesssim 3 \times 10^{-50}\left[\frac{\sec C^{2}}{\sqrt{\mathrm{kg} m}}\right]
$$

This means quite tiny coupling strength.

Open Access. This article is distributed under the terms of the Creative Commons Attribution License (CC-BY 4.0), which permits any use, distribution and reproduction in any medium, provided the original author(s) and source are credited.

\section{References}

[1] Particle Data Group collaboration, K.A. Olive et al., Review of particle physics, Chin. Phys. C 38 (2014) 090001 [inSPIRE].

[2] E.W. Kolb and M.S. Turner, The early universe, Front. Phys. 69 (1990) 1 [InSPIRE].

[3] S. Weinberg, Cosmology, Oxford University Press, Oxford U.K. (2008).

[4] G. Jungman, M. Kamionkowski and K. Griest, Supersymmetric dark matter, Phys. Rept. 267 (1996) 195 [hep-ph/9506380] [INSPIRE].

[5] G. Bertone, D. Hooper and J. Silk, Particle dark matter: evidence, candidates and constraints, Phys. Rept. 405 (2005) 279 [hep-ph/0404175] [INSPIRE].

[6] R.D. Peccei and H.R. Quinn, CP conservation in the presence of instantons, Phys. Rev. Lett. 38 (1977) 1440 [INSPIRE]. 
[7] R.D. Peccei and H.R. Quinn, Constraints imposed by CP conservation in the presence of instantons, Phys. Rev. D 16 (1977) 1791 [INSPIRE].

[8] M. Kuster, G. Raffelt and B. Beltran, Axions: theory, cosmology, and experimental searches, in the proceedings of the $1^{\text {st }}$ Joint ILIAS-CERN-CAST axion training, November 30-December 2, Geneva, Switzerland (2005), Lect. Notes Phys. 741 (2008) 1.

[9] P.W. Graham, I.G. Irastorza, S.K. Lamoreaux, A. Lindner and K.A. van Bibber, Experimental searches for the axion and axion-like particles, Ann. Rev. Nucl. Part. Sci. 65 (2015) 485 [arXiv:1602.00039] [INSPIRE].

[10] CAST collaboration, V. Anastassopoulos et al., New CAST limit on the axion-photon interaction, Nature Phys. 13 (2017) 584 [arXiv:1705.02290] [INSPIRE].

[11] J.E. Kim, Weak interaction singlet and strong CP invariance, Phys. Rev. Lett. 43 (1979) 103 [INSPIRE].

[12] M.A. Shifman, A.I. Vainshtein and V.I. Zakharov, Can confinement ensure natural CP invariance of strong interactions?, Nucl. Phys. B 166 (1980) 493 [InSPIRE].

[13] A.R. Zhitnitsky, On possible suppression of the axion hadron interactions (in Russian), Sov. J. Nucl. Phys. 31 (1980) 260 [Yad. Fiz. 31 (1980) 497] [INSPIRE].

[14] M. Dine, W. Fischler and M. Srednicki, A simple solution to the strong CP problem with a harmless axion, Phys. Lett. 104B (1981) 199 [INSPIRE].

[15] P. Sikivie and Q. Yang, Bose-Einstein condensation of dark matter axions, Phys. Rev. Lett. 103 (2009) 111301 [arXiv:0901.1106] [INSPIRE].

[16] O. Erken, P. Sikivie, H. Tam and Q. Yang, Cosmic axion thermalization, Phys. Rev. D 85 (2012) 063520 [arXiv:1111.1157] [INSPIRE].

[17] K. Saikawa and M. Yamaguchi, Evolution and thermalization of dark matter axions in the condensed regime, Phys. Rev. D 87 (2013) 085010 [arXiv:1210.7080] [INSPIRE].

[18] S. Davidson, Axions: Bose Einstein condensate or classical field?, Astropart. Phys. 65 (2015) 101 [arXiv: 1405.1139] [INSPIRE].

[19] A.H. Guth, M.P. Hertzberg and C. Prescod-Weinstein, Do dark matter axions form a condensate with long-range correlation?, Phys. Rev. D 92 (2015) 103513 [arXiv:1412.5930] [INSPIRE].

[20] S. Weinberg, A new light boson?, Phys. Rev. Lett. 40 (1978) 223 [InSPIRE].

[21] F. Wilczek, Problem of strong $p$ and $t$ invariance in the presence of instantons, Phys. Rev. Lett. 40 (1978) 279 [INSPIRE].

[22] D. Lynden-Bell, Statistical mechanics of violent relaxation in stellar systems, Mon. Not. Roy. Astron. Soc. 136 (1967) 101 [INSPIRE].

[23] C.J. Hogan and M.J. Rees, Axion miniclusters, Phys. Lett. B 205 (1988) 228 [INSPIRE].

[24] E.W. Kolb and I.I. Tkachev, Nonlinear axion dynamics and formation of cosmological pseudosolitons, Phys. Rev. D 49 (1994) 5040 [astro-ph/9311037] [INSPIRE].

[25] S. Davidson and T. Schwetz, Rotating drops of axion dark matter, Phys. Rev. D 93 (2016) 123509 [arXiv: 1603.04249] [INSPIRE].

[26] P.-H. Chavanis, Collapse of a self-gravitating Bose-Einstein condensate with attractive self-interaction, Phys. Rev. D 94 (2016) 083007 [arXiv: 1604.05904] [INSPIRE]. 
[27] C. Beck, Axion mass estimates from resonant Josephson junctions, Phys. Dark Univ. 7-8 (2015) 6 [arXiv: 1403.5676] [INSPIRE].

[28] Y. Kahn, B.R. Safdi and J. Thaler, Broadband and resonant approaches to axion dark matter detection, Phys. Rev. Lett. 117 (2016) 141801 [arXiv:1602.01086] [INSPIRE].

[29] N. Nagaosa and Y. Tokura, Topological properties and dynamics of magnetic skyrmions, Nature Nanotech. 8 (2013) 899.

[30] A.M. Essin, J.E. Moore and D. Vanderbilt, Magnetoelectric polarizability and axion electrodynamics in crystalline insulators, Phys. Rev. Lett. 102 (2009) 146805 [arXiv: 0810.2998] [INSPIRE].

[31] R. Li, J. Wang, X. Qi and S.-C. Zhang, Dynamical axion field in topological magnetic insulators, Nature Phys. 6 (2010) 284 [arXiv:0908.1537] [INSPIRE].

[32] A. Bermudez et al., Wilson fermions and axion electrodynamics in optical lattices, Phys. Rev. Lett. 105 (2010) 190404 [arXiv: 1004.5101] [INSPIRE].

[33] M. Tinkham, Introduction to superconductivity, second edition, Dover Publications, U.S.A. (2004).

[34] N. Kopnin, Theory of nonequilibrium superconductivity, Oxford University Press, Oxford U.K. (2009).

[35] A.A. Abrikosov, On the Magnetic properties of superconductors of the second group, Sov. Phys. JETP 5 (1957) 1174 [Zh. Eksp. Teor. Fiz. 32 (1957) 1442] [INSPIRE].

[36] H.B. Nielsen and P. Olesen, Vortex line models for dual strings, Nucl. Phys. B 61 (1973) 45 [INSPIRE].

[37] A. Schmid, A time dependent Ginzburg-Landau equation and its application to the problem of resistivity in the mixed state, Phys. kondens. Materie 5 (1966) 302.

[38] J. Loudon and P. Midgley, Imaging flux vortices in type II superconductors with a commercial transmission electron microscope, Ultramicroscopy 109 (2009) 700

[39] C.L. Phillips, T. Peterka, D. Karpeyev and A. Glatz, Detecting vortices in superconductors: Extracting one-dimensional topological singularities from a discretized complex scalar field, Phys. Rev. E 91 (2015) 023311.

[40] M. Le Bellac and J.M. Lévy-Leblond, Galilean electromagnetism, Nuovo Cim. B 14 (1973) 217.

[41] G. Blatter et al. Vortices in high-temperature superconductors, Rev. Mod. Phys. 66 (1994) 1125 [INSPIRE].

[42] A. C. Marley, M.J. Higgins and S. Bhattacharya, Flux flow noise and dynamical transitions in a flux line lattice, Phys. Rev. Lett. 74 (1995) 3029.

[43] S. Okuma and N. Kokubo, Vortex dynamics in amorphous $\mathrm{Mo}_{x} S i_{1-x}$ films detected by voltage noise, Phys. Rev. B 61 (2000) 671.

[44] S. Mukerjee and D.A. Huse, Nernst effect in the vortex-liquid regime of a type-II superconductor, Phys. Rev. B 70 (2004) 014506.

[45] P. Sikivie, Experimental tests of the invisible axion, Phys. Rev. Lett. 51 (1983) 1415 [Erratum ibid. 52 (1984) 695] [INSPIRE]. 
[46] S.L. Adler, J. Gamboa, F. Mendez and J. Lopez-Sarrion, Axions and 'Light shining through a wall': a detailed theoretical analysis, Annals Phys. 323 (2008) 2851 [arXiv:0801.4739] [INSPIRE].

[47] A. Aoki and J. Soda, Detecting ultralight axion dark matter wind with laser interferometers, Int. J. Mod. Phys. D 26 (2016) 1750063 [arXiv: 1608.05933] [InSPIRE].

[48] P.S.B. Dev, M. Lindner and S. Ohmer, Gravitational waves as a new probe of Bose-Einstein condensate Dark Matter, Phys. Lett. B 773 (2017) 219 [arXiv:1609.03939] [INSPIRE]. 